\title{
EXTERNAL NOISE EFFECTS IN DOPED SEMICONDUCTORS OPERATING UNDER SUB-THZ SIGNALS
}

\author{
Dominique Persano Adorno, Nicola Pizzolato, \\ DaVide VAlenti and Bernardo SPAGNOLO \\ Dipartimento di Fisica, Group of Interdisciplinary Physics, \\ Università di Palermo and CNISM, \\ Viale delle Scienze, edificio 18, I-90128 Palermo, Italy \\ (e-mails: dominique.persanoadorno@unipa.it,nicola.pizzolato@unipa.it, davide.valenti@unipa.it, \\ bernardo.spagnolo@unipa.it)
}

(Received November 15, 2011 - Revised February 7, 2012)

\begin{abstract}
We study the noise-induced effects on the electron transport dynamics in low-doped n-type GaAs samples by using a Monte Carlo approach. The system is driven by an external periodic electric field in the presence of a random telegraph noise source. The modifications caused by the addition of external fluctuations are investigated by studying the spectral density of the electron velocity fluctuations for different values of the noise parameters. The findings indicate that the diffusion noise in low-doped semiconductors can be reduced by the addition of a fluctuating component to the driving electric field, but the effect critically depends on the features of the external noise source.
\end{abstract}

Keywords: Monte Carlo simulations, fluctuations and noise processes, transport properties.

\section{Introduction}

Semiconductor based devices are always imbedded into a noisy environment that can strongly affect their performance. For this reason, to fully understand the complex scenario of the nonlinear phenomena involved in the devices response, the analysis of noise-induced effects on electron transport dynamics in low-doped n-type samples is very relevant.

Recently an increasing interest has been directed towards the possible constructive aspects of noise on the dynamical response of nonlinear systems. Previous investigations have shown that the addition of external fluctuations to systems, characterized by the presence of intrinsic noise, may affect the dynamics of the system in a counterintuitive way, inducing an enhancement of its stability and resulting in a less noisy response [1-6]. The possibility of reducing the intrinsic noise in semiconductor bulk materials, embedded in a constant electric field in the presence of a Gaussian noise source with a characteristic time, has been theoretically investigated [7]. Detailed studies of the electron transport dynamics in GaAs crystals have revealed that the addition of a correlated fluctuating contribution to a driving sub-terahertz oscillating 
electric field, under specific conditions, can reduce the total noise power $[8,9]$. This result is explained in terms of the noise enhanced stability phenomenon $[10,11]$, arising from the fact that the transport dynamics of electrons in the semiconductor receives a benefit by the constructive interplay between the fluctuating electric field and the intrinsic noise of the system [9]. Furthermore, by considering the integrated spectral density (ISD), which coincides with the variance of the electron velocity fluctuations, a nonmonotonic behaviour as a function of both the amplitude and the correlation time of the external noise source has been found [9, 12].

In this paper we investigate the effects of a different type of external noise on the spectral density on the spectral density of the electron velosity fluctuations. The study is performed by considering a n-doped GaAs semiconductor sample driven by a high-frequency periodic electric field. The electron dynamics is simulated by a Monte Carlo technique which keeps into account all the possible scattering phenomena of the hot electrons in the medium. In particular, we focus on the effect of a non-Gaussian fluctuating contribution to the driving electric field: a random telegraph noise source. This kind of external noise, superimposed to the intrinsic one, is used to tune the dynamic response of the system. Random telegraph (RT) noise is generated by a random process in which the random quantity takes only discrete values and randomly switches between these values. RT noise is observed as two-level or multilevel impulses. Specifically, we report on a preliminary analysis performed by using the two-level random telegraph noise source, that is by considering only fluctuations of equal height, in order to deal with a source of external noise which can be easily generated in practical systems and whose tuning effects are more controllable.

The modifications caused by the addition of external fluctuations are investigated by studying the correlation function of the velocity fluctuations and the integrated spectral density, as a function of the characteristic parameters of the external noise source $[13,14]$. The results are discussed and compared with those obtained in the presence of a correlated noise source. We find the possibility of obtaining a less noisy response in the presence of a driving periodic electric field with random telegraph fluctuations. However, this intrinsic noise reduction is strongly affected by the features which characterize the dichotomous stochastic process, such as the noise level, the frequency of the periodic term and the constant term of the jump rate.

The paper is organized as follows: in Section 2 we present the details of both the physical semiconductor model and the Monte Carlo procedure, together with the statistical quantities used to investigate the electronic noise; in Section 3 the results of our calculations are shown and discussed. Final comments and conclusions are given in Section 4.

\section{Physical model and noise calculation method}

The Monte Carlo code used here follows the procedure described in [15]. The conduction bands of GaAs are the $\Gamma$ valley, four equivalent $L$-valleys and three equivalent $X$-valleys. The parameters of the band structure and scattering mechanisms 
are also reported in [15]. Our code includes the effects of intravalley and intervalley scattering of electrons in multiple energy valleys, and of the nonparabolicity of the band structure. Electron scatterings due to ionized impurities, acoustic and polar optical phonons in each valley as well as all intervalley transitions between the equivalent and nonequivalent valleys are accounted for. We assume field-independent scattering probabilities; accordingly, the influence of the external fields is only indirect through the field-modified electron velocities. All simulations are performed in a GaAs bulk with a free electrons concentration $n=10^{13} \mathrm{~cm}^{-3}$. To neglect the thermal noise contribution and to highlight the partition noise effects we choose a lattice temperature $T=80 \mathrm{~K}$. We assume that all donors are ionized and that the free electron concentration is equal to the doping concentration.

The semiconductor bulk carriers are driven by a fluctuating periodic electric field

$$
E(t)=E_{0} \cos (\omega t+\phi)+\eta(t)
$$

with frequency $f=\omega / 2 \pi$ and amplitude $E_{0} ; \eta(t)$ is the random component of the electric field modeled with a dichotomous stochastic process, characterized by two discrete levels, whose jump rate is a periodic function $\gamma(t)$ which obeys the following equation [16]

$$
\gamma(t)= \begin{cases}0, & \Delta t \leq \tau_{\mathrm{d}}, \\ \gamma_{0}(1+A|\cos 2 \pi v t|), & \Delta t>\tau_{\mathrm{d}} .\end{cases}
$$

Here $\Delta t$ is the the duration of the impulse, i.e. the time interval between two consecutive switches, and $\tau_{\mathrm{d}}$ is the minimum delay between two jumps, that is the time interval after a switch, before another jump can occur. $A$ and $v$ are, respectively, the amplitude and frequency of the periodic term, and $\gamma_{0}$ is the jump rate in the absence of the periodic term. This causes $\eta(t)$ to jump between two values, namely $+D$ and $-D$. The time interval between two consequent pulses and their duration are random, but in all runs we have assumed that the time delay $\tau_{\mathrm{d}}$ of the dichotomous noise is greater than half period $T$ of the deterministic forcing field. Because of the presence of the time delay $\tau_{\mathrm{d}}$ and differently from a standard telegraph noise source, in which the probability of transition depends only on the present state of the system and does not depend on its history [17], our peculiar form of RT noise is not a Markovian process. We have set $A=20$.

The motion of electrons in the presence of an electric field is characterized by an average velocity, which depends on the external parameters of the system, such as the amplitude of the applied field and its frequency. The fluctuations of electron velocity around its mean value correspond to the intrinsic noise of the system. The noise-induced changes of the intrinsic noise properties are investigated by a statistical analysis of the autocorrelation function of the velocity fluctuations and its mean spectral density. Although, due to the presence of the random component, the single excitation is not periodic, our process exhibits cyclostationarity since its average statistical properties vary cyclically with time, i.e. $\langle E(t+m T)\rangle=$ $\langle E(t)\rangle ;\langle v(t+m T)\rangle=\langle v(t)\rangle$, where the brackets $\langle\ldots\rangle$ mean the average over an 
ensemble of different realizations of $E(t)$ and $v(t)$ histories. For systems operating under cyclostationary conditions, the correlation function $C_{\delta v \delta v}(t, \tau)$ of the velocity fluctuations $\delta v(t)=v(t)-\langle v(t)\rangle$ can be calculated [14] as

$$
C_{\delta v \delta v}(t, \tau)=\left\langle v\left(t-\frac{\tau}{2}\right) v\left(t+\frac{\tau}{2}\right)\right\rangle-\left\langle v\left(t-\frac{\tau}{2}\right)\right\rangle\left\langle v\left(t+\frac{\tau}{2}\right)\right\rangle
$$

in which $\tau$ is the correlation time and the average is done over a sequence of equivalent time instants $t=s+m T$, with $s$ belonging to the time interval $[0, T](T$ is the field period) and $m$ is an integer [14]. This two-time symmetric correlation function eliminates any regular contribution and describes only the fluctuating part of $v(t)$. By averaging over the whole set of values of $t$ within the period $T$, the velocity autocorrelation function becomes

$$
C_{\delta v \delta v}(\tau)=\frac{1}{T} \int_{0}^{T} C_{\delta v \delta v}(t, \tau) d t .
$$

According to the Wiener-Kintchine theorem, the spectral density can be calculated as the Fourier transform of $C_{\delta v \delta v}(\tau)$. In the computations of the autocorrelation function we have considered $10^{3}$ possible initial values of $s$ and a total number of equivalent time instants $m \cong 10^{6}$.

If the semiconductor bulk is driven by a static electric field, the shape of the spectral density of electron velocity fluctuations is exclusively determined by the strength of the applied field. In particular, for amplitudes smaller than the threshold field (Gunn field) $E_{\mathrm{G}}$ for intervalley transitions, the diffusion is the most relevant source of intrinsic noise, while, for $E>E_{\mathrm{G}}$, the noise is mainly determined by a partition noise, caused by stochastic carrier transitions between regions characterized by different dynamical properties (intervalley transfers) in momentum space. The partition noise is characterized by a pronounced peak in the spectral density at a frequency $v_{\mathrm{G}}$, which is defined as the "natural" transition frequency of the system between the valleys [18]. In the presence of a high-frequency oscillating field, the noise behaviour depends on both the amplitude and the frequency of the applied field $[9,19]$. In particular, for frequencies $f \geq v_{\mathrm{G}}$, the intervalley transfers are driven by the external field, the system enters in a forced regime of oscillations and the velocity fluctuations become time correlated. In this case, the spectral density is characterized by both a peak centered around the frequency of the periodic signal and a significant enhancement in the low-frequency region [18].

\section{Numerical results and discussion}

As analytically shown in Eqs. 3 and 4 of [7], the electron average velocity is modified by the presence of the external noise. In particular, the most favorable condition to obtain a noise suppression effect in III-V semiconductors is reached when $d^{2} S_{0}(E) / d E^{2}$ is negative and the variance of velocity fluctuations exhibits a maximum. On the base of a preliminary analysis on the behaviour of both the variance of velocity fluctuations and the spectral density $S_{0}(E)$ at zero frequency, 
as a function of the amplitude of the oscillating field (see Figs. 1 and 2 of [9]), we choose a deterministic electric field with amplitude $E_{0}=10 \mathrm{kV} / \mathrm{cm}$ and frequency $f=500 \mathrm{GHz}$. In the absence of external noise, the intensity of the driving field is large enough to allow intervalley transitions from the $\Gamma$ valley to the $L$ valleys $\left(E_{\mathrm{G}}=3.2 \mathrm{kV} / \mathrm{cm}\right)$ and, since the natural transition frequency $v_{\mathrm{G}}$ for our system is $\sim$ $500 \mathrm{GHz}$, the spectral density of electron velocity fluctuations is mainly determined by partition noise $[20,21]$, with the features described in Section 2.

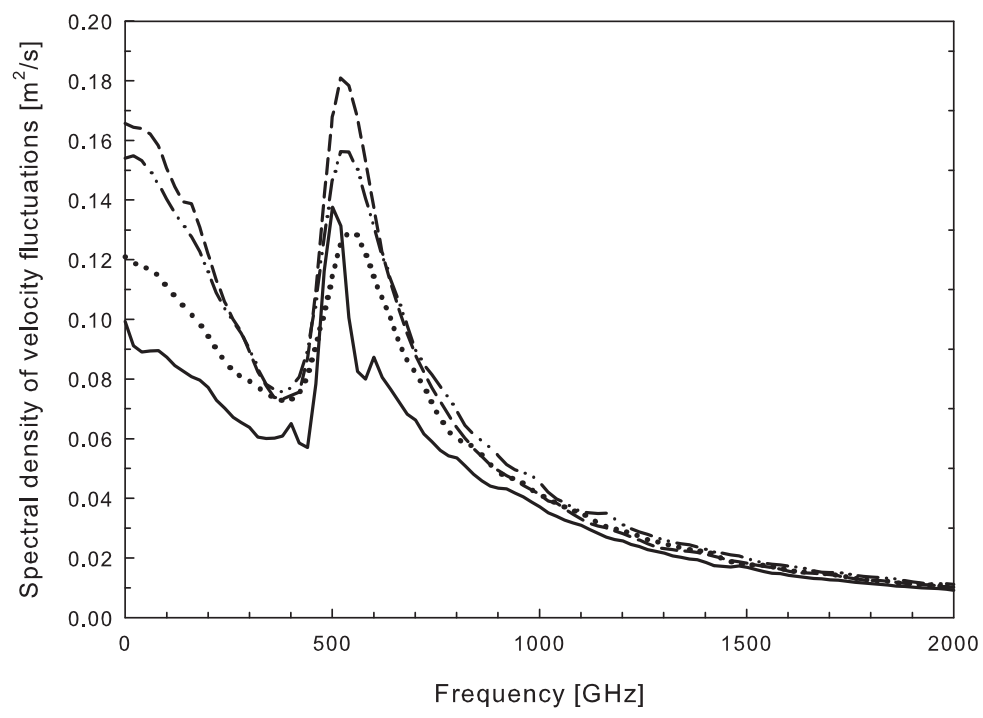

Fig. 1. Spectral density of electron velocity fluctuations as a function of the frequency. $D=4 \mathrm{kV} / \mathrm{cm}$ and $v=500 \mathrm{GHz}$. Dashed line is obtained in the absence of external noise; solid line describes the results obtained with $\gamma_{0}=2 \mathrm{GHz}$; dotted line is obtained with $\gamma_{0}=20 \mathrm{GHz}$; dashed-dotted line is obtained with $\gamma_{0}=200 \mathrm{GHz}$.

In Fig. 1 we show how the spectral density of the electron velocity fluctuations is modified by the presence of an RT source of noise, characterized by $D=4$ $\mathrm{kV} / \mathrm{cm}$ and $v=500 \mathrm{GHz}$, for different values of $\gamma_{0}$, namely $\gamma_{0}=2,20,200 \mathrm{GHz}$. Dashed line, drawn for the comparison purpose, is obtained in absence of external noise. The addition of an RT fluctuating component to the driving electric field strongly affects the spectrum for frequency lower than $1.5 \mathrm{THz}$. In particular, if the frequency $v$ of the periodic term in the jump rate of the RT noise is the same of the driving sub-THz field $(v=f=500 \mathrm{GHz})$, we find changes on both the height of the peak around $500 \mathrm{GHz}$ and the values in the low-frequency region. Specifically, these values critically depend on the value of $\gamma_{0}$. For all the considered values of $\gamma_{0}$, the spectral density of the electron velocity fluctuations in the low-frequency region is strongly reduced and this effect increases with the decrease of $\gamma_{0}$. The behaviour of the spectra, as the parameter $\gamma_{0}$ decreases, resembles that observed in the presence of a correlated noise source with increasing correlation time. However, 
a difference is observed: in the case of RT noise the height of the peak never exceeds the height obtained in the absence of external noise (see Fig. 2(a) in [9]). When the intrinsic noise is mainly due to the partition effect, the height of the peak in the spectral density depends on the populations of the different valleys, reaching a maximum when the populations are nearly at the same level [20, 22]. Since the "effective" electric field experienced by electrons in the presence of a fluctuating field is different, the number of intervalley transitions changes with respect to the case in which the external noise source is absent. This fact, as in the case of a correlated source of noise, can be responsible for the observed changes on both the height of the peak at the resonant frequency and the spectral density values at low frequencies.

At the lowest value of $\gamma_{0}$, the spectrum is characterized by (i) a very narrow peak, the signature of a "strong" forced regime of intervalley transitions, in which velocity fluctuations become time correlated, (ii) a distortion of the curve and (iii) the appearance of two satellite peaks, which could be ascribed to a nonlinear behaviour of the system. The shape of the curve found at the value $\gamma_{0}=2 \mathrm{GHz}$ represents a very interesting result because of its features both in the low-frequency region and at the "resonant" peak, and it needs of further investigations.

The dependence of the intrinsic noise reduction effect on the intensity of the RT noise source has been investigated by studying the integrated spectral density (ISD), that is the total noise power, as a function of the RT noise amplitude, for three different values of $\gamma_{0}$, namely $2 \mathrm{GHz}, 20 \mathrm{GHz}$ and $80 \mathrm{GHz}$. In Fig. 2 the



Fig. 2. External noise spectral density of electron velocity fluctuations as a function of the external noise amplitude. Circles: $\gamma_{0}=2 \mathrm{GHz}$; squares: $\gamma_{0}=20 \mathrm{GHz}$; triangles: $\gamma_{0}=80 \mathrm{GHz} . v=500 \mathrm{GHz}$. 
reduction of the ISD in the presence of external noise is clearly evident for the values of $\gamma_{0}$ up to $20 \mathrm{GHz}$, while the effect of the RT noise is less relevant for higher values of $\gamma_{0}$. The intrinsic noise reduction due to the RT noise is similar to that obtained in the presence of an external source of correlated noise, but it does not show the nonmonotonicity with the noise amplitude found in the case of correlated fluctuations. Moreover, the intrinsic noise suppression obtained in the presence of RT noise is stronger than that found in the presence of correlated noise with the same amplitude [8,9]. From a microscopic point of view, also in this case, the noise reduction can be explained as follows. The fluctuating electric field, by changing the number of intervalley transitions proportionally to the noise amplitude, forces the carriers to visit regions of the momentum space characterized by a smaller variance with respect to the case of zero noise [3]. The behaviour of the ISD curve, for decreasing $\gamma_{0}$, shows that the positive effect of the RT noise is more effective when the jump rate of the stochastic process is lower than the "natural" frequency of the system. With the aim to elucidate how the noise effects depend on the parameters which characterize the jump rate $\gamma(t)$ of the electric field fluctuations, we have calculated (i) the ISD as a function of the RT noise amplitude $D$ for the fixed value $\gamma_{0}=20 \mathrm{GHz}$ and two different values of the frequency of the periodic term, $v=500 \mathrm{GHz}$ and $v=500 \mathrm{THz}$ (see Fig. 3) and (ii) the ISD as a function of $\gamma_{0}$ with $D=4 \mathrm{kV} / \mathrm{cm}$ and $v=500 \mathrm{GHz}$ (see Fig. 4). For a fixed value of $\gamma_{0}$ the intrinsic noise reduction decreases as the frequency $v$ of the function $\gamma(t)$ increases. This circumstance can be ascribed to the fact that, when the frequency $v$ becomes very high, the RT noise switching rate $\gamma(t)$ tends to a constant average value and, as a consequence, the reduction effect becomes smaller. On the other hand, if the frequency $v$ of the periodic term is the same

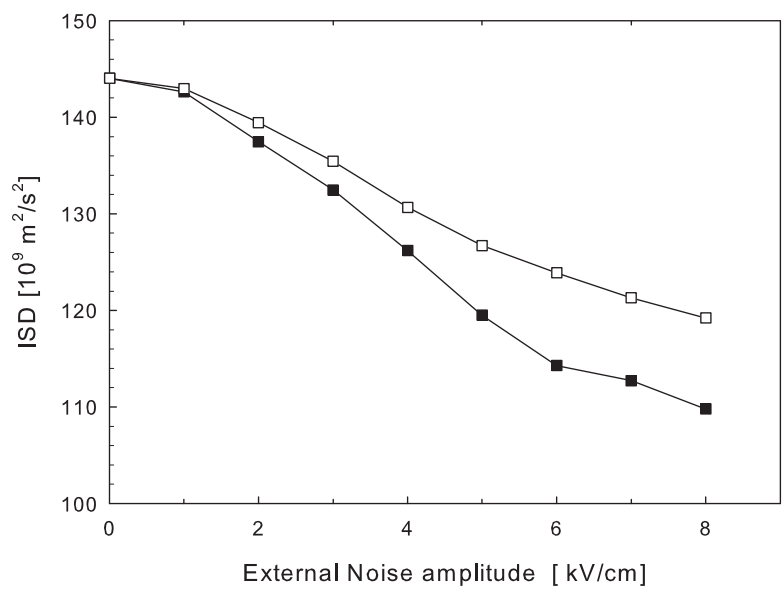

Fig. 3. ISD as a function of the RT noise amplitude obtained with $\gamma_{0}=20 \mathrm{GHz}$ and two different values of the frequency of the periodic term; black squares: $v=500 \mathrm{GHz}$, white squares: $v=500 \mathrm{THz}$. 


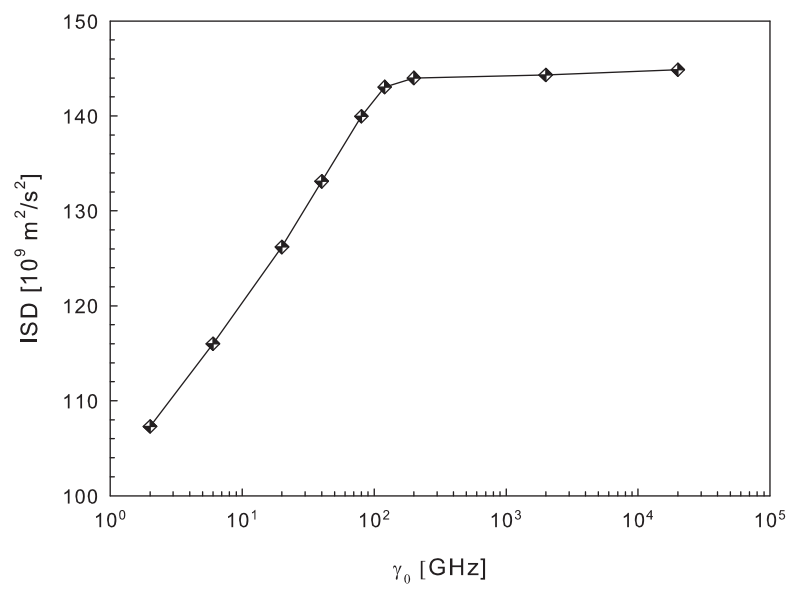

Fig. 4. ISD as a function of the constant term $\gamma_{0}$ obtained with $D=4 \mathrm{kV} / \mathrm{cm}$ and $v=500 \mathrm{GHz}$.

of the driving oscillating field $(v=f=500 \mathrm{GHz})$, the reduction of the intrinsic noise is present for $\gamma_{0}$ values up to $\sim 100 \mathrm{GHz}$, but disappears for higher values, for which the ISD reaches the value obtained in the absence of noise (see Fig. 4). This can be explained by noting that, when the jump rate $\gamma(t)$ becomes too high, the "effective" electric field experienced by the electrons, in the presence of the RT fluctuating field, tends to its deterministic value. In fact, for high values of $\gamma_{0}$ the ISD saturates to the value obtained in the absence of external fluctuations.

\section{Conclusions}

In previous studies the possibility of a suppression of the intrinsic noise in semiconductor bulk materials, subjected to a driving periodic electric field containing time-correlated fluctuations, has been observed. In that case, differently from the static field case, it has been shown that the reduction of the electron velocity fluctuations is not due to an overall effect of intervalley transfers. This is because the velocity variance of an electron moving in the $\Gamma$-valley is reduced by the presence of correlated noise, independently on the transitions to upper valleys, giving rise to longer residence times. This effect can be viewed as a noise enhanced stability (NES) phenomenon [10, 11].

The results reported in this work show that, under specific conditions, the intrinsic noise in n-type GaAs crystals could be also reduced, by adding a two-level RT noise source to the driving high-frequency periodic electric field. These findings have been obtained by numerically investigating the noise-induced modifications of the spectrum of electron velocity fluctuations and the total power of the intrinsic noise. The reduction arises from the fact that the transport dynamics of electrons in the semiconductor receives a benefit by the constructive interplay between the fluctuating 
electric field and the intrinsic noise of the system. The intrinsic noise reduction is strongly affected by the parameters characterizing the dichotomous stochastic process, such as the noise level and the jump rate. In order to highlight on the physical mechanisms responsible for the observed noise reduction and the possible existence of a noise enhanced stability phenomenon, these preliminary results need further investigations. This can be pursued by analyzing the occupation time and the variance of the electron velocity fluctuations in each single energy valley of the semiconductor, performing a detailed study of the correlation functions.

\section{Acknowledgements}

This work was partially supported by MIUR and CNISM.

\section{REFERENCES}

[1] J. M. G. Vilar and J. M. Rubí: Phys. Rev. Lett. 86 (2001), 950-953.

[2] A. Fiasconaro, D. Valenti and B. Spagnolo: Physica A 325 (2003), 136-143.

[3] D. B. Walton and K. Visscher: Phys. Rev. E 69 (8) (2004), 051110.

[4] Y. Seol, K. Visscher and D. B. Walton: Phys. Rev. Lett. 93 (4) (2004), 160602.

[5] N. Pizzolato, A. Fiasconaro, D. Persano Adorno and B. Spagnolo: Physical Biology 7 (5) (2010), 034001.

[6] S. Spezia, D. Persano Adorno, N. Pizzolato and B. Spagnolo: J. Phys.: Condens. Matter 24 (6) (2012), 052204; Acta Phys. B 43 (2012),1191-1201.

[7] L. Varani, C. Palermo, C. De Vasconcelos, J.F. Millithaler, J. C. Vaissiére, J. P. Nougier, E. Starikov, P. Shiktorov and V. Gruzinskis: Is it possible to suppress noise by noise in semiconductors? Proc. of the 4th Int. Conf. Unsolved Problems Noise Fluctuations Phys., Biol., High Technol., 800 pp. 474-479, American Institute of Physics (2005).

[8] D. Persano Adorno, N. Pizzolato and B. Spagnolo: Acta Phys. Pol. A 113 (2008), 985-988.

[9] D. Persano Adorno, N. Pizzolato and B. Spagnolo: J. Stat. Mech., P01039-1-P01039-10 (2009).

[10] N. V. Augdov, B. Spagnolo: Phys. Rev. E 64 (2001), 035102(R).

[11] A. A. Dubkov, N. V. Augdov, B. Spagnolo: Phys. Rev. E 69 (70 (2004), 061103.

[12] D. Persano Adorno, N. Pizzolato and B. Spagnolo: CP1129 Noise and Fluctuations: ICNF 2009, American Institute of Physics (2009), 121-124.

[13] P. Shiktorov, E. Starikov, V. Gruzinskis, L. Reggiani, L. Varani and J. C. Vaissiére: Appl. Phys. Letters 80 (2002), 4759-4761.

[14] T. González, S. Pérez, E. Starikov, P. Shiktorov, V. Gruzinskis, L. Reggiani, L. Varani and J.C. Vaissiére: Microscopic investigaton of large-signal noise in semiconductor materials and devices in Proc. of SPIE 5113 (2003), 252-266.

[15] D. Persano Adorno, M. Zarcone and G. Ferrante: Laser Phys. 10, 310-315 (2000); Laser Phys. 11 (2001), 291-295.

[16] D. Valenti, L. Schimansky-Geier, X. Sailer and B. Spagnolo: Eur. Phys. J. B 50 (2006), $199-203$.

[17] Sh. Kogan: Electronic Noise and Fluctuations in Solids, Cambridge University Press 1996.

[18] D. Persano Adorno, M. C. Capizzo and M. Zarcone: Fluct. Noise Lett. 8 (2008), L11-L22.

[19] D. Persano Adorno, M. C. Capizzo and N. Pizzolato: Acta Phys. Pol. A 113 (2008), 979-984.

[20] P. Shiktorov, E. Starikov, V. Gruzinskis, S. Pérez, T. González, L. Reggiani, L. Varani and J. C. Vaissiére: Phys. Rev. B 67 (2003), 165201-165211.

[21] P. Shiktorov, E. Starikov, V. Gruzinskis, L. Reggiani, L. Varani, J. C. Vaissiere, M. Zarcone, D. Persano Adorno and G. Ferrante: Monte Carlo simulation of electronic noise under large-signal operation in Noise in Physical Systems and 1/f Fluctuations, Ed. Gijs Bosman, World Scientific, pp. 677-680, 2001.

[22] J. P. Nougier: IEEE Trans. Electr. Dev. 41 (1994), 2034-2048. 